\title{
PESQUISAS COM ESTUDANTES DO ENSINO SUPERIOR: ALGUMAS CARACTERÍSTICAS A PARTIR DE PERIÓDICOS NACIONAIS
}

\begin{abstract}
Soely Aparecida Jorge Polydoro Psicóloga e Doutora em Educação pela Universidade Estadual de Campinas. Docente da Faculdade de Educação da Universidade Estadual de Campinas e membro do Grupo de Pesquisa Psicologia e

Educação Superior na mesma instituição.
\end{abstract}

Fernanda Andrade de Freitas Psicóloga e Mestre em Psicologia com ênfase em Avaliação Psicológica pelo Programa de Pósgraduação Stricto Sensu em Psicologia da Universidade São Francisco-Itatiba. Doutoranda pela Faculdade de Educação da Universidade Estadual de Campinas-Unicamp, no Grupo de pesquisa Psicologia e Educação Superior.

\begin{abstract}
Resumo
Esse estudo objetivou levantar características das pesquisas desenvolvidas com os estudantes de ensino superior a partir de periódicos nacionais tendo como foco a Psicologia e a Educação. Para isso, 334 artigos publicados em dois periódicos foram consultados. Dentre eles, 35 foram selecionados, pois indicavam, no resumo e/ou título, o foco no estudante. As categorias analisadas foram: ano de publicação, autoria, referências utilizadas, tipos de pesquisas, e as características dos participantes. Identificou-se que as pesquisas foram, majoritariamente, de natureza descritiva e quantitativa, com autoria múltipla e feminina. Os pesquisadores utilizaram com mais frequência, os artigos como fonte dos estudos. Os estudantes do curso de Psicologia foram os mais estudados, havendo presença de alunos tradicionais e os nãotradicionais na amostra. Esses dados, embora não representativos da literatura nacional, oferecem um panorama que permite discutir algumas tendências e/ou lacunas acerca das variáveis estudadas.
\end{abstract}

Palavras-chave: estudante universitário, ensino superior, pesquisa documental.

\section{RESEARCHES WITH UNIVERSITY STUDENTS: SOME CHARACTERISTICS FROM NATIONAL JOURNALS}

\begin{abstract}
This study aimed to show characteristics from researches developed with university students from national journals focusing Psychology and Education. For that, 334 articles published in two journals were consulted. Among them, 35 were selected, as indicated, in the summary and/or title, students' focus. The categories analyzed were: year of publication, authorship, references used, types of researches and participants' characteristics. It was identified that the research was mainly descriptive and quantitative with multiple author and female. The researchers used frequently the articles as a source of studies. The students of Psychology were the most studied and traditional and non traditional students' were noticed in the sample. These data, despite being unrepresentative of the national literature offer an overview and allow to discuss some trends and/or gaps concerning to the variables studied.

Keywords: university students, college education, documental research.
\end{abstract}

\section{INVESTIGACIÓN SOBRE LOS ESTUDIANTES EN LA EDUCACIÓN SUPERIOR: ALGUNAS CARACTERÍSTICAS DE LA REVISTA NACIONAL}




\begin{abstract}
Resumen
Este estudio tuvo como objetivo la investigación de las características desarrolladas con estudiantes de la educación superior en revistas nacionales centradas en la Psicología y la Educación. Para esto, 334 artículos publicados en dos revistas fueran consultados. Entre ellos, 35 fueron seleccionados como indicado en el resumen y/o titulo, el foco en el estudiante. Las categorías utilizadas fueran: año de publicación, autoría, las referencias utilizadas, los tipos de investigación y las características de los estudiantes. Se identifico que las investigaciones fueron en su mayoría del tipo descriptivo y cuantitativo, con múltiples autores y de mujeres. Los investigadores usaran con más frecuencia los artículos como fuente de estudios. Los estudiantes de Psicología fueran los más estudiados y en la muestra se presentan tradicionales y non tradicionales estudiantes. Estos dados, aunque no representativo de la literatura nacional, ofertas un panorama y permite discutir algunas de las tendencias y/o las lagunas de las variables estudiadas.
\end{abstract}

Palabras clave: estudiantes universitarios, educación superior, investigación documental.

\title{
INTRODUÇÃO
}

Há uma extensa trajetória de produção que demonstra a construção da interface entre a Psicologia e a Educação ao longo dos anos, desde o período colonial. Atualmente, pesquisadores e profissionais tanto da psicologia como da educação têm buscado atuar na direção da superação dos problemas educacionais e criar possibilidades de construção de uma educação efetiva e mais democrática (Antunes, 2003). E, nesta relação, como alerta Bock (2003), é necessário manter a reflexão sobre como "a Psicologia se tornou necessária à educação e como a Psicologia respondeu às demandas que Ihe foram feitas" (p.79). Esta relação entre a Psicologia e a Educação define um campo na Psicologia, denominado Psicologia Educacional ou equivalente. Este campo passa a se constituir como uma identidade própria, o que gera a inserção de disciplinas específicas em cursos de graduação de Psicologia, Pedagogia e demais licenciaturas; a produção de livros e revistas científicas específicos; e a própria organização da associação brasileira de psicologia escolar e educacional ABRAPEE, por exemplo.

Não se pretende, neste artigo, fazer uma descrição sobre a Psicologia Educacional. Destaca-se, porém, que seus objetos de estudos são amplos e os mais diversos, desde os protagonistas da educação como professores, alunos, gestores, funcionários e pais, até as variáveis de natureza educacional e psicológica, como estresse, autoconceito, método de ensino, condições ambientais, desempenho, integração, ética e moral, entre outras. A especificidade dos níveis de ensino é outra variável estudada, a saber, a educação infantil, o ensino fundamental, o médio e o superior. Uma aproximação 
aos desdobramentos de produção no interior de cada um deste níveis pode ser consultado em http://www.inep.gov.br/comped/estudos/.

$\mathrm{Na}$ educação superior, a partir do trabalho de natureza documental organizado por Morosini (2001), intitulado de O "estado do conhecimento" sobre a Educação Superior em Periódicos Nacionais (1968-1995), é possível localizar 15 temáticas estudadas no período de 28 anos, a saber, as políticas públicas de educação, o ensino, a universidade e a sociedade, a avaliação do ensino superior, a história das instituições de ensino superior, a pesquisa, os discentes, a extensão universitária, o corpo docente, a organização acadêmica e a gestão, a manutenção e o financiamento do ensino superior, a autonomia universitária, a relação ensino, pesquisa e a extensão, a natureza jurídica das instituições de ensino superior - IES e corpo o técnico administrativo. Destaca-se que do total das 15 temáticas, o corpo discente foi a sétima maior, representando 5,8\% dos tipos de produção $(n=262)$.

Tendo como base artigos, resumos, notícias, atos normativo e outros tipos de produção, Vasconcelos, Medeiros, Seiffert e Chaves (2001) se debruçaram sobre a comunidade universitária composta por docentes, discentes e técnicoadministrativos. Ao se referirem aos estudantes universitários, as autoras elencaram que as variáveis investigadas foram: o perfil dos estudantes, as atividades por eles realizadas, o acesso à universidade, o desempenho acadêmico, as formas de associação, o movimento estudantil e uma outra categoria que representa assuntos diversos, como perfil de egressos, presença de estrangeiros, compondo a categoria Outros.

Com o intuito de localizar trabalhos cujo foco era o estudante ingressante universitário, Freitas e Polydoro (2009) identificaram 39 trabalhos entre teses e dissertações. As palavras-chave estudadas variaram desde conhecimentos específicos de determinado curso, vivência universitária, processo seletivo, compreensão em leitura, escolha profissional até medidas psicológicas. O público alvo dos trabalhos de pós-graduação eram os ingressantes, provenientes de diferentes cursos, sendo os mais frequentes, os de engenharias, administração, pedagogia e psicologia.

Bariani, Buin, Barros e Escher (2004) pesquisaram em artigos, teses e dissertações de duas instituições paulistas as palavras-chave psicologia escolar educacional e a educação superior. Com base nas pesquisas localizadas, elas selecionaram 34 publicações cujas temáticas envolviam o corpo docente, corpo 
discente e o processo de ensino e aprendizagem. Nessa pesquisa, os alunos provenientes de universidade foram os mais investigados, dado esse que corrobora com os encontrados por Oliveira et al (2007) e Witter (1996). Para Bariani, Buin, Barros e Escher (2004), as variáveis investigadas com os alunos diziam respeito à depressão, ansiedade, funções de leitura, perfil sociométrico, motivações, expectativas, conhecimentos e opiniões, desempenho dos alunos em leitura, relações interpessoais, entre outras. Para as autoras, a maioria das pesquisas (59\%) foi desenvolvida em apenas um curso, e quanto aos turnos dos cursos frequentados, é pequeno o número de estudos realizados com alunos matriculados em curso noturno (12\%). Elas afirmaram que, a maioria dos alunos pesquisados eram iniciantes ou concluintes, o que dificilmente viabiliza entender o processo de mudança ocorrida com os alunos no decorrer da graduação. Especialmente dada a sua complexidade que, como destacam Pascarella e Terenzini $(1991,2005)$, as mudanças podem ser elas de natureza acadêmica, pessoal, profissional, entre outras.

Para Bariani et al (2004), dentre as 34 pesquisas lidas, apenas uma delas utilizou o delineamento experimental. A estratégia mais utilizada para coletar informações foi a aplicação de questionários, em relação às entrevistas e outras técnicas. Já, Oliveira e colaboradores (2007), ao analisar as publicações em avaliação psicológica no contexto educacional em sete periódicos brasileiros, evidenciaram que os testes psicométricos eram os mais utilizados em detrimento dos questionários/entrevistas, situações problemas, observação, técnicas projetivas entre outras.

A partir da análise dos 304 trabalhos inscritos na categoria Escola/Educação, no I Congresso Brasileiro de Psicologia: Ciência e Profissão, Santos, Oliveira, Joly e Suehiro (2003) categorizaram 15 temáticas focalizadas. Elas apontaram que a categoria Ensino Superior ficou entre as cinco temáticas mais frequentes, permanecendo atrás das categorias: Orientação Profissional, Formação do Educador, Necessidades Especiais e Outras. Em outro estudo, Oliveira, Cantalice, Joly e Santos (2006) demonstraram que o Ensino Superior é o nível escolar mais estudado pelos pesquisadores, no periódico Psicologia Escolar e Educacional.

Essas pesquisas mencionadas anteriormente, de forma geral, remetem às pesquisas desenvolvidas nacionalmente. Em contraponto, Witter (1996) investigou a leitura no contexto universitário, tendo como base dois periódicos 
internacionais. Assim, ela levantou 57 amostras investigadas nas pesquisas, 44 pertenciam ao estudo do estudante universitário, 10 eram voltadas aos calouros, e outros três envolviam o estudante de pós-graduação $(n=2)$ e professores $(n=1)$. Ela concluiu, dentre outros aspectos, que as pesquisas apresentaram uma metodologia avançada, $72,5 \%$ delas mostraram delineamento experimental.

Ainda, no contexto internacional, ressalta-se que há uma preocupação em produzir estudos de revisão sobre a produção a respeito do ensino superior, o que tem gerado diversas publicações de natureza documental. O conjunto destas publicações sintetiza décadas da literatura sobre a experiência do estudante durante sua formação na educação superior. São destaques, por ordem cronológica, os trabalhos de Feldman e Newcomb, 1969; Bowen, 1977; Strange, 1994; Terenzini e Pascarella, 1991, 2005. Por um lado, este conhecimento produzido indica que o estudante experiência uma rede ampla e integrada de mudanças que inclui aspectos de natureza cognitiva e não cognitiva, e por outro, destaca o papel das características e oportunidades institucionais neste processo, o que tem gerado modelos teóricos que representam a complexa relação de variáveis entre estudantes e instituição na trajetória de formação, bem como, recomendações para a prática educativa e de gestão (ver, por exemplo, Kuh, 2009 \& Reason, 2009).

Diferentemente deste quadro, no Brasil ainda são poucas as revisões nacionais sobre o tema. Fato que é agravado, pois nem sempre os alunos de ensino superior participantes dos estudos, são de fato o público-alvo. Em alguns trabalhos, os estudantes são selecionados pelo critério de conveniência por parte dos pesquisadores devido à proximidade com a amostra (Oliveira \& et al, 2006). Associado a isso, e ao papel da interface Psicologia e Educação na análise das questões envolvidas na experiência de formação no ensino superior, esse trabalho objetivou identificar as características das pesquisas recentes desenvolvidas com estudantes de ensino superior relatadas em periódicos nacionais com foco na relação Psicologia e Educação.

\section{MÉTODO}

Considerando os critérios estabelecidos para a obtenção dos dados era a produção de revistas científicas nacionais da Psicologia Educacional sobre o estudante de ensino superior, foram analisados os títulos dos periódicos apresentados no relatório Qualis (2009 - http://www.anpepp.org.br/dir- 
2008/avaliacao/2009-QUALIS\%20PSICOLOGIA-CLASSIFICACAO.xls). Apenas duas revistas preenchiam o critério de interface Psicologia e Educação, a saber: Psicologia da Educação (http://www.pucsp.br/pos/ped/revista) e Psicologia Escolar e Educacional (http://www.abrapee.psc.br/sumarios.htm). Trata-se de periódicos com distribuição semestral e o período consultado para a realização do levantamento foi de 2000 a 2009.

Foram consultados todos os números de ambos os periódicos no período indicado, preferencialmente no formato impresso e, quando necessário, no formato on-line, e selecionadas as pesquisas que tinham presente em suas amostras o estudante de ensino superior. A seleção dos artigos se deu a partir do título e do resumo. Após a seleção do material, foi realizada uma releitura de todos os títulos e resumos para a categorização, conforme o ano de publicação, autoria (única ou múltipla e sexo), as referências utilizadas (artigos, livros, teses, dissertações e outros), os tipos de pesquisas, segundo o tratamento dos dados (qualitativa ou quantitativa) e o delineamento (descritiva ou experimental). As características dos participantes (número de participantes, idade média, gênero, curso e momento do curso) e os temas de interesse foram também levantados, sendo todos os dados, inseridos em uma planilha eletrônica para viabilizar as análises de conteúdo e descritiva.

\section{RESULTADOS E DISCUSSÃo}

Do universo de 334 artigos publicados em ambos os periódicos no período de 2000 a 2009, apenas 35 (10,4\%) tinham o estudante do ensino superior pertencente à amostra. Destes, 32 eram da revista Psicologia Escolar e Educacional e três da revista Psicologia da Educação. A seguir será apresentada a frequência dos artigos selecionados conforme o ano e a revista.

Observa-se que o periódico Psicologia Escolar e Educacional, no período de nove anos, apresentou mais estudos tendo o estudante do ensino superior como participantes, comparado ao periódico Psicologia da Educação. Essa diferença pode ser explicada pelo fato do periódico Psicologia Escolar e Educacional apresentar mais trabalhos empíricos. Além disso, nota-se que, com exceção do ano de 2001, há uma contínua presença desta temática ao longo dos anos de publicação da revista, indicando uma acentuação do interesse a partir do ano de 2005. 
Tabela 1.

Frequência dos artigos por ano em cada periódico.

\begin{tabular}{ccc}
\hline Revistas & $\begin{array}{c}\text { Psicologia Escolar e } \\
\text { Educacional }\end{array}$ & $\begin{array}{c}\text { Psicologia da } \\
\text { Educação }\end{array}$ \\
Anos & 3 & 0 \\
2000 & 0 & 0 \\
2001 & 2 & 1 \\
2002 & 2 & 0 \\
2003 & 3 & 0 \\
2004 & 5 & 0 \\
2005 & 5 & 1 \\
2006 & 4 & 1 \\
2007 & 5 & 0 \\
2008 & 3 & Não disponível \\
2009 & 32 & 3 \\
Total & & \\
\hline
\end{tabular}

No que tange à autoria dos trabalhos, foram identificados 53 autores, sendo 37 pesquisadoras e 16 pesquisadores. Observa-se que as mulheres são mais presentes que os homens, o que evidencia a feminilidade nas publicações na área da Psicologia e Educação, conforme demonstram outros estudos (Oliveira \& et al, 2006, 2007). Dos 35 artigos localizados, seis são de autoria única, sendo todas mulheres. As autorias múltiplas variavam entre dois a seis autores, sendo mais frequente a de dois pesquisadores, presentes em 16 artigos.

Ainda referente à autoria, das 35 pesquisas, 30 são de autoria de pesquisadores nacionais e 5 internacionais. Esse dado evidencia a interlocução entre diferentes países, a saber, Bélgica, Espanha e Portugal, e reitera a necessidade do intercâmbio. Todos os trabalhos internacionais foram publicados no periódico Psicologia Escolar e Educacional que, em 2007 houve uma edição especial somente com pesquisas internacionais, tendo em um dos artigos, estudantes do ensino superior, como participantes.

A identificação das palavras-chave do título do artigo permitiu identificar o interesse dos pesquisadores ao estudar aspectos sobre o estudante de ensino superior e sua experiência de formação. Foram observados as seguintes categorias temáticas: aspectos de natureza pessoal, tais como, criatividade, estilos cognitivos, motivação, hábitos e expectativas, processo de adaptação, autoconceito, capacidade cognitiva e metas acadêmicas dos estudantes; aspectos de natureza vocacional, como por exemplo, escolha profissional, interesses profissionais e a inserção no mercado de trabalho; e aspectos de 
natureza acadêmica, como a discussão das estratégias de aprendizagem, os métodos de estudo e o rendimento. A partir da descrição das palavras-chave nota-se que, na amostra selecionada, as variáveis mais exploradas estão voltadas para aspectos de natureza pessoal do estudante, em detrimento dos aspectos de natureza vocacional e acadêmica. O rol de variáveis estudadas nos artigos selecionados parecem menos amplas ao se comparar com as encontradas por Vasconcelos, Medeiros, Seiffert e Chaves (2001), diferença essa provavelmente justificada pela amostra selecionada.

Quanto à natureza da pesquisa em relação aos seus objetivos, dos 35 resumos identificados, 31 se preocuparam em descrever o fenômeno sem nele interferir, caracterizando-se como pesquisas descritivas e, em apenas dois resumos, o foco era avaliar o desempenho do aluno após a sua intervenção, indicando delineamento de natureza experimental. Não foi possível identificar esta característica em dois dos resumos. A tendência de poucas pesquisas experimentais desenvolvidas na área da Psicologia é apontada por alguns autores (Campos, 2008), em contraponto à realidade internacional (Witter, 1996). Esse dado nos faz refletir acerca da formação do psicólogo, seja na graduação como também na pós-graduação, e o seu impacto na área de pesquisa (Werneck, 2006; Luna, 2007).

Acerca das análises dos dados descritos nos resumos dos artigos, foi possível identificar que em 25 os pesquisadores optaram por análises quantitativas, em quatro, por análises qualitativas como o uso de técnicas de grupo focal e o uso de narrativas e, em dois trabalhos, a análise foi mista. Vale ressaltar que em quatro resumos não foi possível identificar essa informação.

As pesquisas científicas têm o levantamento bibliográfico como uma das etapas do trabalho científico, que se justifica, entre outras coisas, em identificar as lacunas e as implicações para futuras pesquisas. Nesse sentido, das 35 pesquisas que tem como amostra os estudantes do ensino superior, foi utilizada a média de 29,7 referências por artigo $(D P=10,8)$, variando de 8 a 54 referências. A partir da Tabela 2, observa-se que as referências no formato de artigos científicos foram as mais utilizadas. A categoria Outros engloba relatórios científicos e/ou referências cuja natureza não foi possível identificar. 
Tabela 2.

Estatísticas descritivas acerca das referências utilizadas nos artigos.

\begin{tabular}{lcccc}
\hline Tipo de publicação & Mínimo & Máximo & Média & Desvio Padrão \\
\hline Artigos científicos & 0 & 32 & 15,11 & 9,06 \\
Livros & 1 & 25 & 10,23 & 6,33 \\
Anais de Eventos & 0 & 13 & 1,63 & 2,70 \\
Dissertações & 0 & 3 & 1,17 & 1,17 \\
Teses & 0 & 6 & 0,80 & 1,30 \\
Outros & 0 & 4 & 0,77 & 1,16 \\
\hline
\end{tabular}

O número de estudantes do ensino superior que compunham as amostras dos estudos foi outra categoria analisada. Como foram utilizadas as informações disponíveis nos resumos dos artigos, em nove estudos não foi possível identificar o número de participantes, já que não estava mencionado no resumo. Tendo como base os outros resumos, observou-se que a média do número de estudantes do ensino superior que participaram das pesquisas foi de $M=117,71$ $(D P=150,56)$, tendo o mínimo de 8 e o máximo de 632 participantes. A quantidade de participantes é uma informação relevante, mas é pertinente levar em consideração a natureza das pesquisas e a análise dos dados adotada pelos pesquisadores, pois a quantidade está relacionada ao delineamento do estudo e implica na generalização dos resultados obtidos nas pesquisas (Campos, 2008).

Algumas características localizadas nos resumos e/ou títulos, acerca dos estudantes do ensino superior serão discutidas. Quanto ao momento do curso, apesar da informação não constar de 15 dos resumos estudados, observou-se que os ingressantes $(n=10)$ foram mais destacados nos resumos que os estudantes de semestres intermediários $(n=5)$, que por sua vez, foi maior que os concluintes $(n=4)$. Uma das 35 pesquisas sinalizou no resumo que tinha os egressos do curso de Fisioterapia como participantes.

A partir dos títulos e/ou resumos, verificou-se que há a presença de alunos dos mais diferentes cursos de formação envolvidos nos estudos. Observa-se que os estudantes do curso de Psicologia foram os mais presentes, conforme Tabela 3. Os dados corroboram os encontrados por Freitas e Polydoro (2009). Em três dos resumos dos 35 artigos estudados não constava a informação sobre o curso de origem dos estudantes. 
Tabela 3.

Frequência do curso de origem dos estudantes participantes dos estudos.

\begin{tabular}{lcc}
\hline Cursos & $F$ & $\%$ \\
\hline Psicologia & 10 & 31,2 \\
Administração & 5 & 15,6 \\
Engenharia & 4 & 12,5 \\
Pedagogia & 3 & 9,3 \\
Medicina & 3 & 9,3 \\
Direito & 3 & 9,3 \\
Nutrição & 1 & 3,2 \\
Fisioterapia & 1 & 3,2 \\
Educação Física & 1 & 3,2 \\
Ciência da Computação & 1 & 3,2 \\
\hline Total & 32 & 100 \\
\hline
\end{tabular}

Ainda, acerca dos cursos de formação, é possível constatar que, em duas pesquisas há presença de estudantes provenientes de três cursos diferentes; em quatro pesquisas foram consultados estudantes de dois cursos, e na maioria $(n=19 ; 31,2 \%)$ há a presença de alunos de apenas um curso. Em 10 dos resumos, não foi possível identificar essa informação. Esse dado aponta a tendência do pouco investimento em coletar informações com alunos provenientes de cursos diferentes, seja por questões burocráticas, financeiras e/ou pelo tempo despendido para localização e aprovação por parte das Instituições de Ensino Superior e/ou pelo Comitê de Ética em Pesquisa (CEP). A decisão pelo número de cursos envolvidos na coleta de dados sobre o estudante e sua vivência no ensino superior deve ser decorrente do problema de pesquisa levantado e não por questões burocráticas e limites de acesso à população de interesse.

Outro dado levantado foi a idade dos participantes. Dos 35 resumos lidos, em apenas oito deles constava a idade dos participantes. Em cinco a idade mínima e a máxima se faziam presentes, sendo que nos outros três, era indicada apenas a média de idade. A média da idade citada nos resumos variou de 18 a 38,8 anos. A amplitude destes valores permite observar que tanto o aluno tradicional como o não-tradicional (nesse caso, o critério para indicar ou não a tradicionalidade dos estudantes do ensino superior foi a idade - aluno-adulto, muito embora, hajam outras características que podem ser levadas em consideração, como por exemplo, ser o primeiro curso ou não) estavam 
presentes nos estudos. Por meio da análise qualitativa nota-se que os alunos tradicionais estão presentes nos estudos em que, os pesquisadores coletaram informações com alunos de curso integral como Medicina e Engenharia, por exemplo, ou com alunos ingressantes. Já, os alunos não-tradicionais são encontrados em estudos que têm presente alunos do curso noturno, ou no qual há comparação entre ingressantes ou concluintes. A identificação destas características reforça os dados apontados em diferentes Censo da Educação Superior (INEP, 2004, 2008, por exemplo) sobre a diversificação da população estudantil do ensino superior, assim como, em função disso, a importância de se pesquisar as especificidades e aspectos condicionais das variáveis envolvidas nesta heterogeneidade.

\section{CONSIDERAÇÕES FINAIS}

Para identificar algumas características das pesquisas desenvolvidas com os alunos do ensino superior, foram consultados os resumos de dois periódicos nacionais, que assumem a interface entre a Psicologia e a Educação. Dado o atendimento à especificidade do critério de seleção referente à interface entre Psicologia e Educação do período a ser estudado, deve-se destacar que os dois periódicos analisados não são representativos de todas as revistas nacionais em que os estudantes do ensino superior estão presentes, fato já amplamente sinalizado por outros estudos documentais (Morosini, 2001). Por outro lado, é possível confrontar os dados obtidos deste estudo com outros divulgados.

Nesse sentido, observa-se algumas tendências e/ou confirmações como a feminilidade das publicações, a autoria múltipla (Oliveira et al, 2006, 2007), a tendência de apenas um curso ser mais explorado em detrimento de outros (Bariani, Buin, Barros \& Escher, 2004; Freitas \& Polydoro, 2009) e o pouco desenvolvimento de pesquisas experimentais (Bariani et al, 2004; Campos, 2008). As lacunas também podem ser reafirmadas, como a necessidade de se investir mais em pesquisas com alunos do curso noturno e o aluno adulto.

Não se pode encerrar este texto sem destacar as características dos títulos e resumos das produções científicas normalmente observadas em nossa literatura. É sabido que os títulos e resumos devem conter as informações mais relevantes dos trabalhos e elementos que caracterizam o seu conteúdo, para que o leitor, ao localizá-los, possa decidir por sua leitura integral ou não. Percebe-se, no entanto, que estas recorrentes recomendações não são atendidas. Como 
refere Ferreira (2002), sob aparente homogeneidade que as normas aplicariam ao resumo, há grande heterogeneidade, explicada pela interação da representação de cada autor sobre este gênero discursivo e as exigências do meio de divulgação utilizado. Para a autora, a análise de determinada produção científica por meio dos resumos pode, no máximo, produzir uma das possíveis histórias desta produção.

Todavia, a partir deste estudo, constata-se que a Psicologia Educacional tem oferecido sua contribuição à Educação Superior, mesmo que envolta de algumas lacunas e tendências. Por outro lado, evidencia-se outras possibilidades de investigação, como explorar experiências de outros cursos de formação, como os tecnológicos; particularidades dos alunos adultos e/ou trabalhadores; o impacto de implantação de programas e intervenções; e análises estatísticas do tipo modelagem dos dados, viabilizando a produção de modelos teóricos sobre a vivência do processo de formação no ensino superior.

\section{REFERÊNCIAS}

Antunes, M. A. M. (2003). Psicologia e Educação no Brasil: Um olhar históricocrítico. In M. E. M Meira \& M. A. M. Antunes (Orgs.), Psicologia Escolar: Teorias críticas (pp. 138-168). São Paulo: Casa do Psicólogo.

Bariani, I. C. D., Buin, E., Barros, R. C., \& Escher, C. A. (2004). Psicologia Escolar e educacional no ensino superior: análise da produção científica. Psicologia Escolar e Educacional, 8(1), 17-28.

Bock, A. M. B. (2003). Psicologia da Educação: cumplicidade ideológica. In M. E. M Meira \& M. A. M. Antunes (Orgs.), Psicologia Escolar: Teoria, críticas (pp. 79-103). São Paulo: Casa do Psicólogo.

Bowen, H. R. (1977). Goals: the intended outcomes of higher education (in) Investment in Learning: The Individual and Social Value of American Higher Education. San Francisco: Jossey-Bass.

Campos, L. F. L. (2008). Métodos e Técnicas de Pesquisa em Psicologia. Campinas: Alínea.

Feldman, K.; Newcomb, T. (1969). The impact of college on student. San Francisco. Jossey-Bass.

Ferreira, N. S. A. (2002). As pesquisas denominadas "estado da arte". Educação \& Sociedade, 79, ano XXIII, 13-21. 
Freitas, F. A., \& Polydoro, S. A. J. (2009). Estudo sobre ingressantes no ensino superior disponível no portal da capes. In Resumos de Comunicação Científicas, IX Congresso Nacional de Psicologia Escolar e Educacional: Construindo uma prática profissional na educação para todos ( $\mathrm{s} / \mathrm{p}$ ). São

Paulo: Associação Brasileira de Psicologia Escolar e Educacional.

INEP - Instituto Nacional de Estudos e Pesquisas Educacionais (2004). Censo da

Educação Superior de 2004. Recuperado em 04/12/2008, do Instituto Nacional de Estudos e Pesquisas Educacionais

http://www.inep.gov.br/superior/censosuperior/sinopse/.

INEP - Instituto Nacional de Estudos e Pesquisas Educacionais (2008). Censo da Educação Superior de 2008. Recuperado em 28/06/2009 do Instituto Nacional de Estudos e Pesquisas Educacionais http://www.inep.gov.br/superior/censosuperior/sinopse/.

Kuh, G. D. (2009). What student affairs professionals need to know about student engagement. Journal of College Student Development 50(6), 683706.

Luna, S. V. (2007). Planejamento de pesquisa: uma introdução. São Paulo: EDUC.

Morosini, M. C. (2001). Introdução. Em M. C. Morosini (org.). Educação Superior em periódicos nacionais (1968-1995). Brasília: MEC/Inep/Comped. Recuperado em 05/01/2010, do Instituto Nacional de Estudos e Pesquisas Educacionais

http://www.inep.gov.br/download/cibec/2001/estado do conhecimento/ed ucacao superior periodicos nacionais.pdf.

Oliveira, K. L., Santos, A. A. A., Noronha, A. P. P., Boruchovitch, E., Cunha, C. A., Bardagi, M. P., \& Domingues, S. F. S. (2007). Produção científica em avaliação psicológica no contexto escolar. Psicologia Escolar e Educacional 11(2), 239-251.

Oliveira, K. L., Cantalice, M. C., Joly, M. C. A. R., \& Santos, A. A. A. (2006) Produção científica de 10 anos da revista Psicologia Escolar e Educacional (1996/2005). Psicologia Escolar e Educacional 10(2), 283-292.

Pascarella, E. T., \& Terenzini, P. T. (1991). How collage affects students: a third decade of research. San Francisco: Jossey-Bass.

Pascarella, E. T., \& Terenzini, P. T. (2005). How collage affects students: a third decade of research. San Francisco: Jossey-Bass. 
Qualis (2009). Qualis de Periódicos da Área de Psicologia. Recuperado em 21/01/2010. http://www.anpepp.org.br/dir-2008/avaliacao/2009QUALIS\%20PSICOLOGIA-CLASSIFICACAO.xIs.

Readon, R. D. (2009). An examination of persistence research through the lens of a comprehensive conceptual framework. Journal of College Student Development, 50(6), 659-682.

Santos, A. A. A., Oliveira, K. L., Joly, M. C., \& Suehiro, A. B. (2003). I Congresso Nacional de 'Psicologia - Ciência e Profissão': O que tem sido feito na psicologia educacional. Psicologia Escolar e Educacional, 7(2), 135-144.

Strange, C. (1994). Student development the evolution and status of an essential idea. Journal of College Student Development,35(6), 399-412.

Vasconcelos, H. C., Medeiros, L. G. M., Seiffert, O. M. L. B., \& Chaves, V. L. J. (2001). A comunidade universitária. In M. C. Morosini (Orgs.), Educação Superior em periódicos nacionais (1968-1995). Brasília: MEC/Inep/Comped. Recuperado em 04/01/2010, do Instituto Nacional de Estudos e Pesquisas Educacionais

http://www.inep.gov.br/download/cibec/2001/estado do conhecimento/ed ucacao superior periodicos nacionais.pdf.

Werneck, V. R. (2006). Sobre o processo de construção do conhecimento: O papel do ensino e da pesquisa. Ensaio: Avaliação Política Pública Educacional, 14(5), 173-196.

Witter, G. P. (1996). Avaliação da produção científica sobre leitura na universidade (1989/1994). Psicologia Escolar e Educacional, 1(1), 31-37.

Contato: ferfreitas@ig.com.br

Recebido em: 10/03/2010

Revisado em: 04/05/2010

Aceito em: 16/06/2010 